\title{
Gathering and synthesizing information during the development of user re- quirements and engineering specifications
}

\section{Ibrahim Mohedas, University of Michigan}

Ibrahim Mohedas is currently a Ph.D. candidate in mechanical engineering at the University of Michigan. His research focuses on the design of medical devices for resource limited settings, particularly related to the use of design ethnography in developing these technologies. He received his B.S. in mechanical engineering from the University of Texas at Austin in 2011.

\section{Dr. Shanna R. Daly, University of Michigan}

Shanna Daly is an Assistant Research Scientist and Adjunct Assistant Professor in the College of Engineering at the University of Michigan. She has a B.E. in Chemical Engineering from the University of Dayton and a Ph.D. in Engineering Education from Purdue University. Her research focuses on idea generation, design strategies, design ethnography, creativity instruction, and engineering practitioners who return to graduate school. She teaches design and entrepreneurship courses at the undergraduate and graduate levels. Her work is often cross-disciplinary, collaborating with colleagues from engineering, education, psychology, and industrial design.

\section{Prof. Kathleen H. Sienko, University of Michigan}

Kathleen H. Sienko is a Miller Faculty Scholar and Associate Professor of Mechanical and Biomedical Engineering at the University of Michigan (UM). She earned her Ph.D. in 2007 in Medical Engineering and Bioastronautics from the Harvard-MIT Division of Health Science and Technology, and holds an S.M. in Aeronautics \& Astronautics from MIT and a B.S. in Materials Engineering from the University of Kentucky. She directs both the Sensory Augmentation and Rehabilitation Laboratory (SARL) and the Laboratory for Innovation in Global Health Technology (LIGHT). SARL focuses on the design, development, and evaluation of medical devices, especially for balance-impaired populations such as individuals with vestibular loss or advanced age. LIGHT focuses on the co-creative design of frugal innovations to address healthcare challenges in resource-limited settings. Prof. Sienko has led efforts at the University of Michigan to incorporate the constraints of global health technologies within engineering design at the undergraduate and graduate levels. She is the recipient of a CAREER Award from the National Science Foundation, a Teaching Innovation Prize from the UM Provost, and a UM Undergraduate Teaching Award. While at MIT, she was a winner of the MIT \$50K Entrepreneurship Competition. 


\section{Gathering and synthesizing information during the development of user requirements and engineering specifications}

\section{Introduction}

The development of user requirements is a key component of the front-end design phases and can have significant impact on product success ${ }^{1}$. Well-defined user requirements have been cited as a pivotal aspect of software development where work on effective requirements elicitation has been performed most extensively ${ }^{2}$. In addition to software development, the medical device industry has begun to emphasize the importance of interacting with end-users during the development of user requirements and engineering specifications in order to reduce medical mistakes caused by user errors ${ }^{3}$. In fact, the FDA now requires developers to interact more directly with their end-users during the design process in order to prevent 'use-related hazards ${ }^{3}$. Recently, research has also looked at the importance of eliciting accurate user requirements for medical devices in order to increase their safety and usability ${ }^{5-7}$. While the critical nature of user requirements within design has been the focus of previous and on-going research, the manner in which students learn the process of developing user requirements has not been well studied. Thus, we sought to understand how students use information sources during the development of user requirements and engineering specifications.

\section{Background}

The development of user requirements requires the designer to gather, synthesize, and use extensive amounts of information and data. In some cases, design projects are an engineering student's first significant introduction to this type of advanced information processing. Design communication documents are similar to explanatory writing in that one must analyze multiple information sources and subsequently make decisions based upon this analysis. Furthermore, designers must deal with conflicting information, questionable data sources, and advanced technical topics.

The front-end phases of design (problem definition, development of user requirements, and translation to engineering specifications) require a particularly large amount of information processing because one is just beginning to understand the design problem and must gain a deep understanding of the stakeholders who may be affected by the product's design. Developing user requirements requires knowledge from multiple disciplines and engineering students must be prepared to use both technical and non-technical skillsets during this phase of design ${ }^{9}$. In addition, the fluid and changing nature of requirements runs contrary to engineering students' previous coursework where one correct solution generally exists ${ }^{10}$.

Comparisons of expert versus novice designers can also help us understand the design processes in which students have the most difficulty. Studies have found that novices typically spend less time gathering information than do expert designers, and that experts spend more time during the problem scoping phase of design ${ }^{11}$. Furthermore, final design quality for engineering students has been correlated to the time students spent defining the problem they were attempting to solve; those who spent more time defining and refining the problem tended to produce higher quality designs $^{12}$. In light of the fact that expert designers spend more time than novices gathering information in order to better define the design problem, research is necessary to 
understand how students engage in information gathering, and what effect this information gathering has on students' design decisions.

This study sought to address this knowledge gap by investigating how students gather, synthesize, and use information when developing user requirements and engineering specifications. Understanding how students process information gathered during design may allow instructors to counter the common mistakes students make when developing user requirements and engineering specifications. Furthermore, research in this topic outside of software engineering is needed to determine whether the lessons learned in software development can apply to other disciplines, such as medical device design.

\section{Research Design}

Study Purpose

The following research questions motivated this study:

- How do students' plans compare with their execution of the front-end phases of design with respect to gathering, synthesizing, and using information?

- Which information sources do students rely on most when developing user requirements and engineering specifications?

- Why do students choose particular information or information sources during front-end design?

\section{Participants}

The study tracked six design teams as they developed user requirements and engineering specifications for their senior capstone design projects. Each design team had four students. Four teams consisted exclusively of mechanical engineering students and two teams each had one biomedical engineering student in addition to the mechanical engineering students. All students were enrolled in the same capstone design course. The design teams focused on the design of global health technologies for low-income settings (i.e., diagnostic devices, training devices, and medical support equipment).

\section{Data Collection}

As part of their design course, students were asked to develop a plan for how they would generate user requirements and engineering specifications for their projects. In this assignment design teams listed all the information sources they planned to use, explained how the user requirements would potentially be affected by the information sources, and justified why the information sources were important. During the following weeks students developed user requirements and engineering specifications for their first design report and subsequently refined these requirements for their second design report. These three documents (the plan, the first design report, and the second design report) comprised the documented data that was collected for the research study. Subsequently, semi-structured interviews were conducted with four of the design teams. During these interviews, teams were asked to comment on how and why their plans differed from their actual approach. Interviews were transcribed for data analysis. The Institutional Review Board of the University of Michigan approved this study, and informed consent was obtained from all subjects before interviews began. 


\section{Data Analysis}

The three written documents were qualitatively coded using the following methodology. First, all information sources that design teams used were identified. These were grouped into eleven general and exhaustive categories (as seen in Figure 2). Second, information sources were tallied for each user requirement in order to determine: 1) the total number of distinct information sources teams planned to use versus the number they actually used to develop user requirements and engineering specifications, and 2) the types of information sources that contributed to each user requirement/engineering specification pair. The interview transcripts were qualitatively analyzed using an iterative inductive coding strategy to identify the prominent themes that emerged during the interviews ${ }^{13}$. Established guidelines for theme identification were used to analyze the data ${ }^{14}$. Coding was iterated until changes ceased to be made to themes.

\section{Results}

To answer our first research question, we compared the average number and standard deviation of information sources students planned to consult per user requirement/engineering specification pair with the average number and standard deviation of information sources they actually used per user requirement/engineering specification pair in the teams' design reports. The results are presented in Figure 1. The number of information sources students planned to consult was consistently greater than the actual number of information sources that students used to develop user requirements and engineering specifications. Furthermore, all teams used, on average, less than two information sources per user requirement/engineering specification pair (average for all teams was 1.5 information sources per user requirement/engineering specification pair). One can see from Figure 1 that students planned to consult a much larger number of information sources than they ended up using. This indicates that while they may have seen value in using varied information sources, they did not implement this strategy into their design projects.

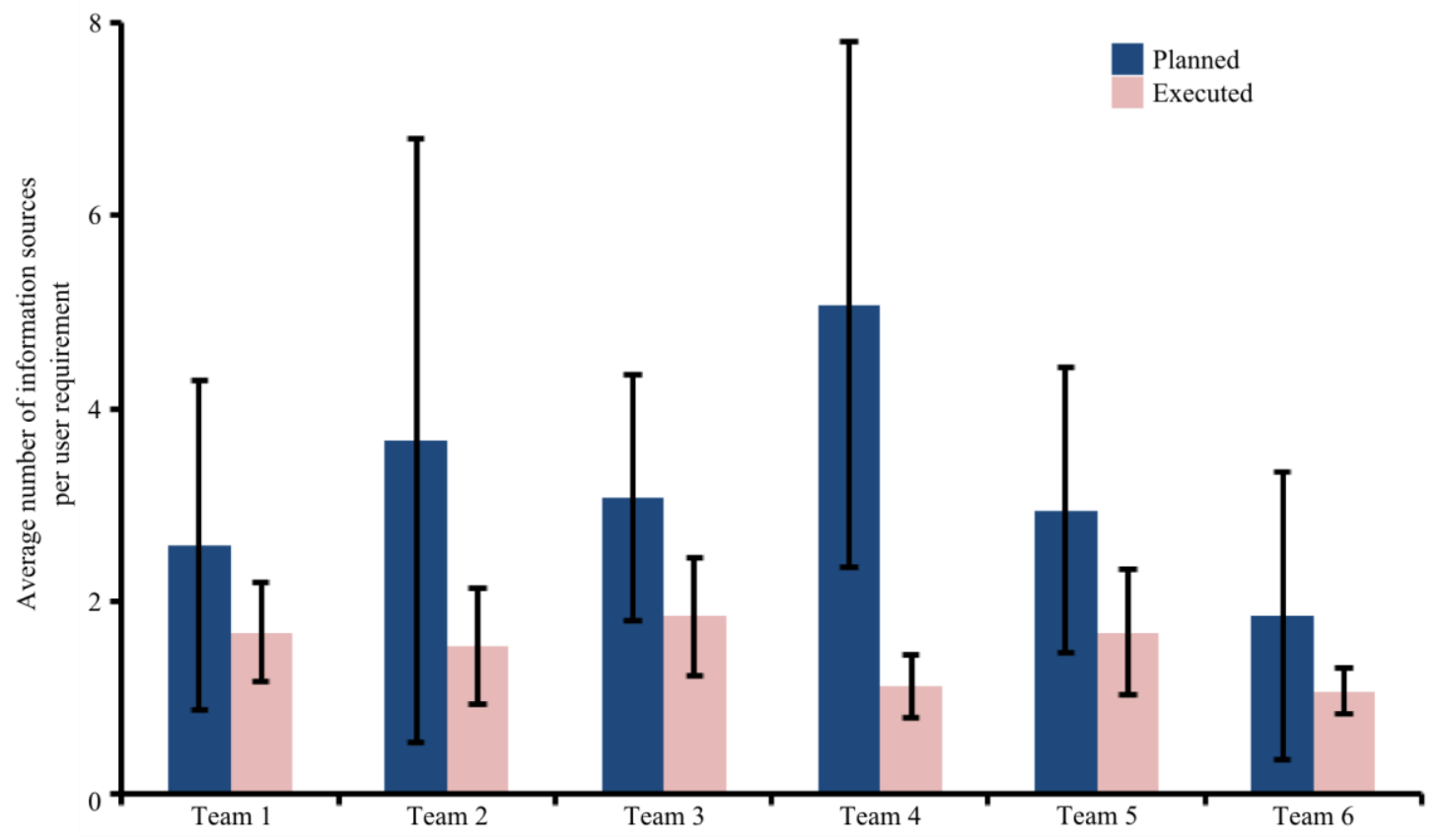

Figure 1: Average number of distinct information sources students planned to use and then used per user requirement; error bars indicate the standard deviation. 
Our second research question sought to determine which information sources students relied upon most heavily. The qualitative analysis of the students' plans and design reports provided the total number of instances of when each information source was mentioned. These results are displayed in Figure 2. The categories of information sources in Figure 2 are exhaustive; i.e., students did not cite any information sources not captured in the categories in Figure 2. Certain information sources featured heavily in the plans that teams developed but then did not heavily influence their actual user requirements or engineering specifications. Most noticeably, end-users (or proxy end-users) as well as other stakeholders featured extensively in the design teams' plans, but were almost completely absent from their design reports. Furthermore, information sources in the three categories of research, benchmark/patents, and online resources were all used more extensively in the development of user requirements/engineering specifications than the students had planned.

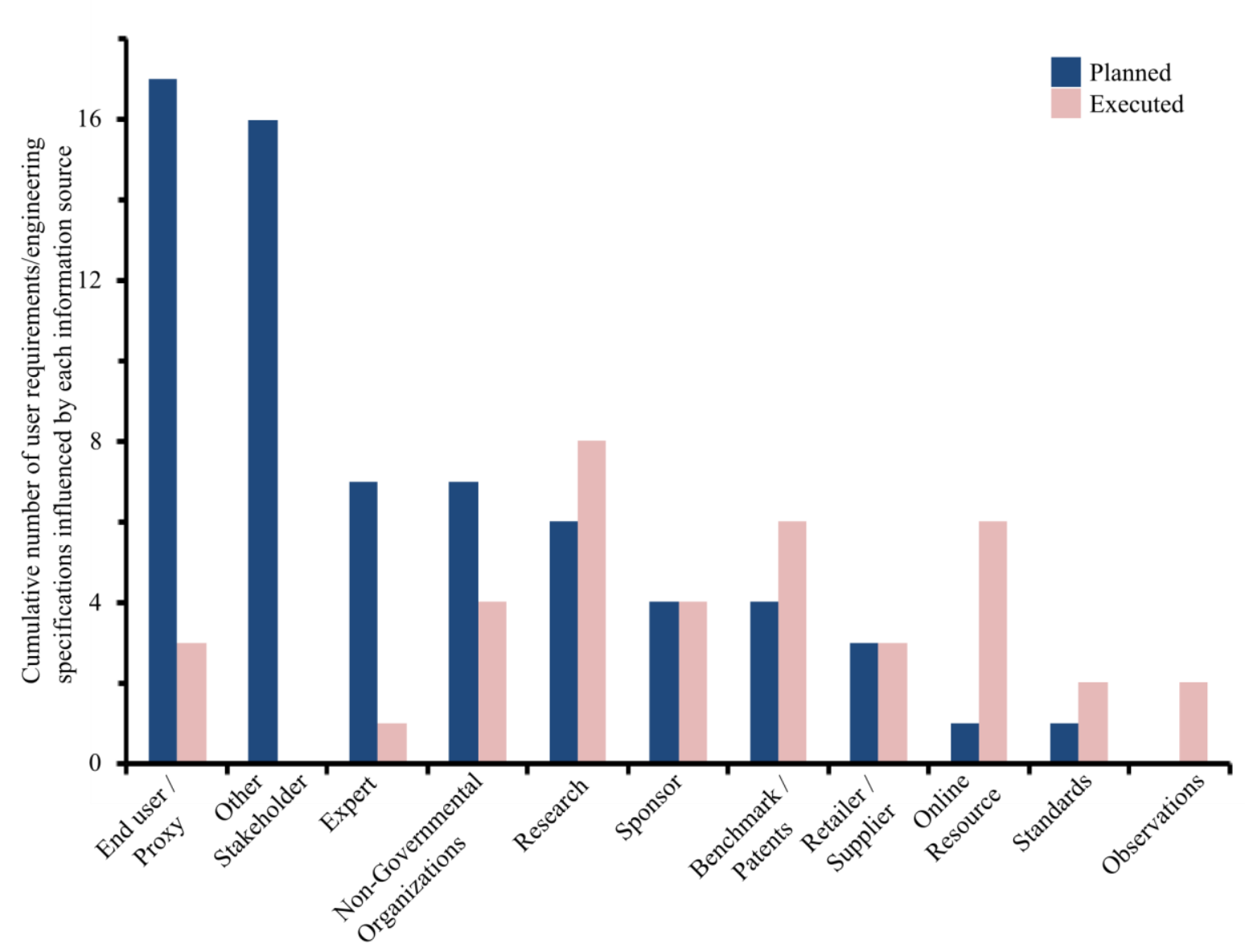

Figure 2. Cumulative number of information sources students planned to and consulted during the development of user requirements and engineering specifications. 
Our final research question sought to understand why students emphasized particular information sources when developing user requirements and engineering specifications. Several reasons emerged during the semi-structured interviews.

One cause of students' reduced use of end-users and other stakeholders as information sources was students' view that end-users and other stakeholders were most useful towards the end of the design process, during product validation. One team described how they would consider interacting with end-users once they had a prototype developed and were ready for product testing.

"The big thing is testing, we don't have access to [end-users] because that requires a lot of approval. [The end-users] wouldn't help us develop the [concepts], but once we [have] a prototype, they [will] help us refine... some of the requirements."

This team did not consider that speaking with end-users could have supplied them with valuable information. Later on in the semester, the team did speak with end-users and received negative responses to their design concept, forcing them to drastically redesign. Pursuing a design concept that was unwanted by end-users could have been prevented had the students engaged end-users in the development of the user requirements and engineering specifications during the early part of the semester. Perceiving end-users and other stakeholders as important only in product evaluation could have contributed to the significant reduction in use of these information sources.

Another contributing factor to the reduction in end-users and other stakeholders as information sources for user requirements and engineering specifications is students' perception of the appropriateness of these information sources. During interviews, design teams spoke to the fact that interviews did not represent reliable information sources and that academic literature or benchmarking were more appropriate. One team described this during their interview.

"[For] a lot of [the user requirements] we had support from observations or interviews but felt that we needed literature to...secure it and back it up."

The team made a similar comment later on indicating how literature was a better information source than interviews.

"...We cited the literature, since it was just more concrete than [an] interview."

The team quoted above had performed extensive observations and interviews in order to define their project and develop user requirements. However, they emphasized that academic literature represented a better source for developing user requirements and engineering specifications. Other teams may have had similar perceptions on the use of interview data and this may have caused them to discount these information sources altogether. While design teams should be encouraged to seek verification within academic literature and benchmarking, this shouldn't cause them to abandon other sources of information entirely. 


\section{Discussion}

Ideally, student teams would consult a variety of information sources and synthesize this information in order to develop user requirements and engineering specifications. This would lead to an iterative process where user requirements and engineering specifications are refined and changed as more information and multiple perspectives are introduced. A review of industry experts in requirements engineering demonstrated that graduating engineers need this type of experience in order to be prepared to begin design work within an industry setting ${ }^{10}$.

Within this study, however, we found that students planned to consult many information sources when developing user requirements and engineering specifications, but in practice they tended to rely on a much smaller number of sources and active synthesis of information was not commonly performed. On average, teams used 1.5 information sources per user requirement/engineering specification pair that they developed. The discrepancy between plans and execution of user requirements and engineering specifications indicates that students faced too many challenges when synthesizing information or deemed it to be unnecessary in order to develop effective requirements.

It was also noted that some planned information sources were much more likely to be abandoned during the development of user requirements and engineering specifications than others. For example, end-users and other stakeholders featured prominently within the design teams plans, however, they were largely unused when actually developing user requirements and engineering specifications. Interviews revealed that students viewed end-users and stakeholders primarily as a means for testing and evaluating their products and that interviews and observations were not as appropriate as benchmarking and academic literature. Changing this perception through the use of real world case studies that demonstrate the vital nature of end-users and stakeholders during the product development process may help to counter students' current views and push them to incorporate more varied perspectives into their design process.

We note two limitations to this study. First, design teams within this study worked on the design of medical devices, a field with which most engineering students have limited experience. This may have added complexity to the task of developing user requirements and engineering specifications and may have contributed to the teams' use of a more limited number of information sources. Furthermore, the end-users of medical devices (doctors, nurses, patients, etc.) may not have been as easily accessible as the end-users of typical consumer products and may have contributed to design team's minimal use of end-users and other stakeholders. Second, the design teams may have thought they were only supposed to cite their most reliable source of information in their design reports. Therefore, synthesis may have occurred but was simply not reflected in the students' writing. Future studies will address these limitations by expanding beyond medical device design teams and expanding the interview protocol to have students explicitly state all information sources consulted (regardless of their presence in the design reports). Future studies will also attempt to isolate confounding variables, such as time pressure and difficulty accessing information sources, that may have prevented students from gathering and synthesizing multiple information sources while developing user requirements and engineering specifications. 


\section{Conclusion}

This study focused on assessing students as they gathered, synthesized, and used information to develop user requirements and engineering specifications. While students originally planned to perform extensive gathering and synthesis, in the end they only referred to a small number of information sources for each user requirement/engineering specification pair. This discrepancy indicates that while they may see theoretical value in consulting a variety of information sources, in practice, they encounter too many challenges or constraints to implement these plans. Interviews revealed that certain information sources (such as end-users and stakeholders) may be ignored due to a perception that they are inferior. Correcting this misperceptions and demonstrating how user requirements and engineering specifications are improved through the incorporation of multiple information sources is key to improving students' ability to perform front-end design. Furthermore, capstone and other design courses can demonstrate that even if multiple information sources may not always agree, it is the designer's job to synthesize the information until a coherent user requirement and engineering specification is developed.

\section{Acknowledgments}

This work was supported by the University of Michigan's Rackham Merit Fellows program, the National Science Foundation's Graduate Research Fellowship program, the National Science Foundation's Research Initiation Grants in Engineering Education, the National Science Foundation's CAREER program (RAPD-0846471, funded under the American Recovery and Reinvestment Act of 2009), and the University of Michigan Center for Research on Learning and Teaching's Investigating Student Learning Grant. The study team thanks Grace Cravens and Kristen Ydoate for their help with data collection and organization, and the senior design students who participated in this study.

\section{Bibliography}

1. Dieter GE. Problem Definition and Need Identification. In: Engineering Design.Vol 15.; 2009:75-115.

2. Jones C. Patterns of Software Failure and Success. Thomson; 1996.

3. Walsh T, Beatty PCW. Human factors error and patient monitoring. Physiol Meas. 2002;23(3):R111-32.

4. Guidance for Industry and FDA Premarket and Design Control Reviewers - Medical Device Use-Safety: Incorporating Human Factors Engineering into Risk Management. FDA. 2011.

5. Martin JL, Clark DJ, Morgan SP, Crowe J a, Murphy E. A user-centred approach to requirements elicitation in medical device development: a case study from an industry perspective. Appl Ergon. 2012;43(1):184-90.

6. Martin JL, Murphy E, Crowe J a, Norris BJ. Capturing user requirements in medical device development: the role of ergonomics. Physiol Meas. 2006;27(8):R49-62.

7. Garmer K, Ylv J, Karlsson ICM. User participation in requirements elicitation comparing focus group interviews and usability tests for eliciting usability requirements for medical equipment : a case study. Int $J$ Ind Ergon. 2004;33:85-98.

8. Gause D, Weinberg G. Exploring Requirements: Quality BEFORE Design. New York: Dorste House; 1989.

9. Nuseibeh B, Easterbrook S. Requirements Engineering: A Roadmap. In: International Conference on Software Engineering.Vol 1. Ireland; 2000:35-46. 
10. Macaulay L, Mylopoulos J. Requirements engineering: An educational dilemma. Autom Softw Eng. 1995;2(4):343-351.

11. Atman CJ, Adams RS, Cardella ME, Turns J, Mosborg S, Saleem J. Engineering Design Processes: A Comparison of Students and Expert Practitioners. J Eng Educ. 2007;96(4):360 - 379.

12. Sobek DK, Jain VK. Relating Design Process to Quality: A Virtual Design of Experiments Approach. $J$ Mech Des. 2007;129(5):483.

13. Borrego M, Douglas EP, Amelink CT. Quantitative, Qualitative, and Mixed Research Methods in Engineering Education. J Eng Educ. 2009;98(1):53-66.

14. Creswell J. Research design: Qualitative, quantitative, and mixed methods approaches. Thousand Oaks, CA: Sage Publications; 2003. 